\title{
Improvement of Radial basis Function Interpolation Performance on Cranial Implant Design
}

\author{
Ferhat Atasoy \\ Computer Engineering Department \\ Karabuk University, Karabuk, Turkey \\ Baha Sen \\ Computer Engineering Department \\ Ankara Yildirim Beyazit University, Ankara, Turkey
}

\author{
Fatih Nar \\ Computer Engineering Department \\ Konya Food and Agriculture University, Konya, Turkey
}

\author{
Ismail Bozkurt
Neurosurgery Department
kiri State Hospital, Cankiri, \\ Ismail Bozkurt
Neurosurgery Department
iri State Hospital, Cankiri, Tut \\ Ismail Bozkurt
Neurosurgery Department
Cankiri State Hospital, Cankiri, Turkey
}

\begin{abstract}
Cranioplasty is a neurosurgical operation for repairing cranial defects that have occurred in a previous operation or trauma. Various methods have been presented for cranioplasty from past to present. In computer-aided design based methods, quality of an implant depends on operator's talent. In mathematical model based methods, such as curvefitting and various interpolations, healthy parts of a skull are used to generate implant model. Researchers have studied to improve performance of mathematical models which are independent from operators' talent. In this study, improvement of radial basis function (RBF) interpolation performance using symmetrical data is presented. Since we focused on the improvement of $\mathbf{R B F}$ interpolation performance on cranial implant design, results were compared with previous studies involving the same technique. In comparison with previously presented results, difference between the computed implant model and the original skull was reduced from $7 \mathrm{~mm}$ to $2 \mathrm{~mm}$ using newly proposed approach.
\end{abstract}

Keywords-Cranioplasty; interpolation on medical images; radial basis function interpolation; symmetrical data

\section{INTRODUCTION}

Cranioplasty is a neurosurgical operation for repairing cranial defects that have occurred in a previous operation or trauma. This operation is important for both aesthetics and health [1]. Encephalitis, cerebritis, trauma, malignancy, hydrocephalus, epilepsy, mental or psychological disorders are associated with cranial bone defects [2], [3]. The main goals of cranioplasty are protection of intracranial contents and providing normal development and growth of the brain in children [4].

Various metals, ceramics, synthetic materials can be used for cranioplasty. The task is to complete the damaged skull bone with the selected material. Cranioplasty operations are performed on frontal bone, parietal bone, occipital bone, sphenoid bone, and portion of the temporal bone [1].

The oldest cranial operations dates back to 7000 B.C. in ancient Egypt [1], [5]. Archaeological finds indicate that inorganic materials have been used much earlier than organic materials. Bones were used for cranioplasty from a wide population of donor groups such as rib bone and tibia, in the

This study was supported by Ankara Yildirim Beyazit University as prescientific research project with 587 project number. 19th century. Although many different materials and methods have been described up to now, there is no consensus on which method is better [1].

An ideal implant material must have following features for cranioplasty applications [1], [5]:

- It must close and fit the defected part of the skull completely

- Not dilated with heat

- Resistance to infections

- Radiolucency

- Lightweight and compatible with tissues (biocompatibility)

- Easy to shape

- Ready to use

- Not expensive

- Resistant to biomechanical procedures

- Easily sterilized

- Non-inflammatory and non-carcinogenic

Thickness of implant varies according to the material. Therefore, implant mold should be specially created for implant material. While surface interpolation may be a good choice to manufacture titanium implant, it may not be right choice for cement-based materials such as methacrylate.

Computer-aided manufacturing of cranial implants have come into use with increasing processing speed of computers and development on imaging and modeling. In previous studies, implants were created with mathematical model or using solid modeling software.

Carr et al. designed cranial implants with radial basis function (RBF) based surface interpolation method on computed tomography (CT) images. In the study, they began with detection of defected part of skull and a height map was created for the defected part and nearby. Unknown areas (greater values on height map) were computed with RBF by 
using known values of neighbors (obtained from non-damaged part of the skull). The results were computed with various radial kernels and thin-plate spline was specified as the optimal kernel for cranial implant design [6].

Heissler et al. designed titanium implants for the defected part by using CAD/CAM according to the anatomical structure on the healthy (symmetrical) side of a skull. In this study, healthy side of the skull was mirrored and the mirrored data was applied for defected part of the skull. 12 male and 3 female patients between 21 and 35 years old were treated clinically and only one of the implants was removed due to premature infection. Reason of infection was interpreted as a corner of the implant may not be fully placed [7].

Lee et al. made manipulations on CT images to perform simulation, segmentation and planning processes. They used polymethylmethacrylate material in their work and developed a rapid prototyping device. The subject of the study was an 8year-old boy. There is a large defect on his skull's left side. In the study, mirrored image of healthy side of the skull was used and a device was developed which can quickly produce implants as a result of the obtained data [8].

Fu et al. used multi-point forming and reverse engineering techniques for implant design. They used arithmetical profile curve blending method based on a well-proportioned point cloud data acquired with analyzing the patients' CT images. They produced titanium implant model for new points by using multiple point forming pressure machine [9].

Gerber et al. designed patient-specific cranial implants with low-cost material polymethylmethacrylate by using computer aided design method. Despite low-cost and widespread use of polymethylmethacrylate material, the technique is a timeconsuming method. Thus, surgery time and risk of infection increase. In this study, implant manufacturing and operation time was shortened by using computer aided implant design method. Primarily, a mold was created with the patient's CT data by using 3D printer. Then, the implant was produced by using the mold and it was implanted to the patient. Three patients were treated successfully using the proposed technique [10].

Yusoff et al. created a 3D model from 2D CT images. In this study, they called the technique as biomedical model and used the model in pre-surgery planning. The manufactured model was produced in 45 hours and 19 minutes depending on the size and complexity. Entire skull was modeled with the manufactured bio-model as the manufactured bio-model provided educational usage and testing before surgical operations [11].

Kun et al. designed implants by using OpenCV and OpenGL object viewer interactively. A user selected symmetry plane on 2D CT images and defected region completed with symmetrical data. Then, OpenCV filled the defected region with symmetrical contour. At the end, the user modified the implant because of asymmetry of the skull [12].

Castelan et al. studied modeling and manufacturing technique of an implant. They manufactured a bio-model of a skull by a 3D printer and modeled an implant using Solidworks software. Titanium was preferred as the implant material in the study since its high-strength characteristic. In the study, symmetrical data and CAD software were used to create the implant [13].

In proposed method by Rudek et al., a missing region of a skull was defined by curvature descriptors. They applied optimization technique of artificial bee colony to estimate descriptor parameters. The estimated descriptors were searched in database to replace defected region. Thus, an implant was modeled from similar images automatically [14].

Van der Meer described a technique to design a cranial implant for all sort of defects. In the study; process, material selection, design, and production were fully controlled by a user. He used Geomatic Studio 12 for data conversion from dicom to surface model and filled holes by curvature-based filling on the software [15].

In general, an operator uses CAD/CAM software to design an implant and symmetrical data is used to complete defected region of a skull. If the operator is skilled, implant will be wellfitted and aesthetically successful. As a conclusion, success of implant manufacturing depends on the operator's talent. Mathematical model based methods are robust but they must be finely-modeled. However, since skull is not completely symmetric and symmetrical data is not always available, studies that use only symmetrical data are not robust. RBF is one of the mathematical models using known neighbors of data-free regions of a skull.

In this study, previously presented scattered data estimation for cranioplasty applications with RBF [16] has been improved. For this purpose, implant shape was created by a mathematical model successfully for high-strength materials such as titanium. Obtained results are smoother and outer surface of the implant is similar to original. This is important in terms of the social and psychological status of the patients. The model has still disadvantages such as inner surface interpolation error and quality of results depends on symmetrical data, symmetry plane position and calculation of normal vector of scattered data.

\section{METHOD}

\section{A. RBF Based Interpolation}

Completion process of cranial defect is obviously a 3D curve fitting or scattered data estimation problem. The estimated data must be in accordance with non-damaged neighbors' bone data geometrically. RBF is one of the most frequently used modern approach to complete data-free regions. This approach is convenient when the problem depends on the multivariate or multi-parameters of scattered data. Besides, it is an appropriate estimation of scattered data in high dimensional space [6], [16], [17].

RBF approach is generally defined as [6], [12]:

$$
f: \mathbb{R}^{d} \rightarrow \mathbb{R}
$$

The real-valued function $f$ of $d$ variables is approximated by $s: \mathbb{R}^{d} \rightarrow \mathbb{R}$. Here, given values $\left\{f\left(x_{i}\right): 1=1,2, \ldots, n\right\}$, where $\left\{x_{i}: 1,2, \ldots, n\right\}$ is a set of discrete points in $\mathbb{R}^{d}$, are called the nodes of interpolation. Thus, approximation form of interpolation is achieved as: 


$$
\begin{gathered}
s(x)=p_{m}(x)+\sum_{i=1}^{n} \lambda_{i} \varnothing\left(\left\|x-x_{i}\right\|\right), \\
x \in \mathbb{R}^{d}, \lambda_{i} \in \mathbb{R}
\end{gathered}
$$

where, $p_{m}$ is low-degree polynomial, or does not exist, ||. || is Euclidean norm, and $\emptyset$ stands for a fixed function from $\mathbb{R}^{+}$ to $\mathbb{R}$. Radial basis function $s$ is a linear combination of translates of the single radially symmetric function $\emptyset(\|\|$.$) ,$ plus a low-degree polynomial. Space of all polynomials of degree at most $m$ in $d$ will be indicated by $\pi_{m}^{d}$. Afterwards, the coefficients $\lambda_{i}$ of the approximation $s$ are calculated by assuming that $s$ fulfills the interpolation conditions as

$$
s\left(x_{j}\right)=f\left(x_{j}\right), \quad j=1,2, \ldots, n
$$

with the side conditions:

$$
\sum_{j=1}^{n} \lambda_{j} q\left(x_{j}\right)=0, \text { for all } q \in \pi_{m}^{d}
$$

Some popular RBF kernels are given as:

$\emptyset(r)=r \quad$ Linear
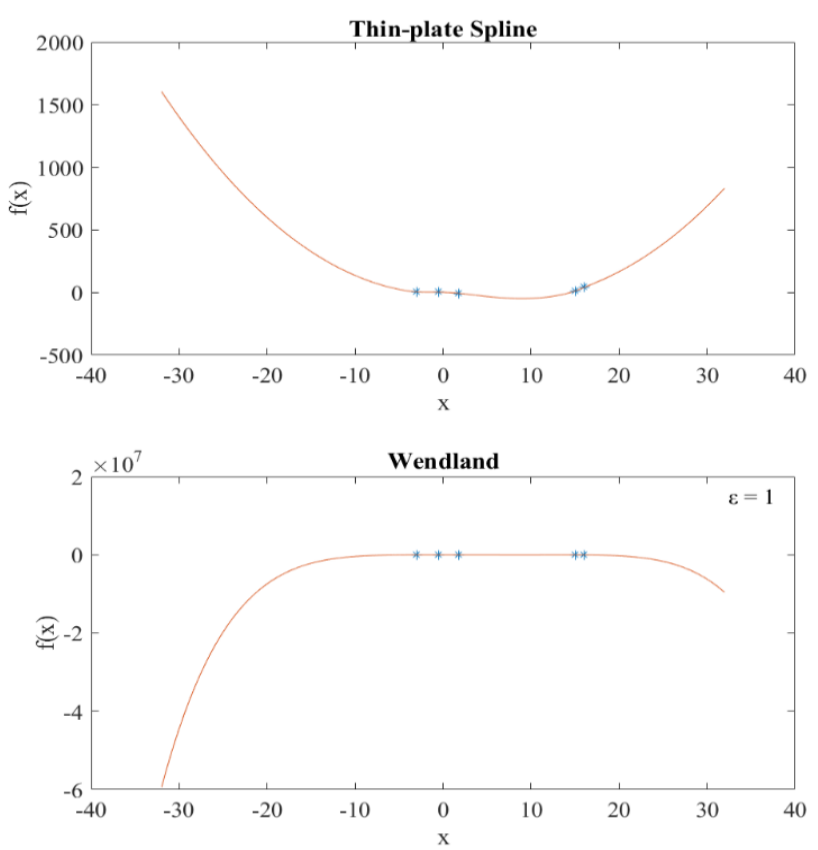

Fig. 1. The obtained curves of various RBF kernels.

In $2 \mathrm{D}$ space, RBF method is applied as the following [6], [16], [17]:

$$
\left[\begin{array}{cc}
A & Q \\
Q^{T} & 0
\end{array}\right]\left[\begin{array}{l}
\lambda \\
C
\end{array}\right]=\left[\begin{array}{l}
f \\
0
\end{array}\right]
$$

where

$$
\begin{gathered}
A=\left(a_{i j}\right)=\left(\varnothing\left(\left\|x_{i}-x_{j}\right\|\right)\right) \\
Q=\left[\begin{array}{ccc}
1 & x_{1} & y_{1} \\
1 & x_{2} & y_{2} \\
\vdots & \vdots & \vdots \\
1 & x_{n} & y_{n}
\end{array}\right]
\end{gathered}
$$

$\varnothing(r)=r^{2} \log r \quad$ Thin-plate spline

$\emptyset(r)=e^{-\alpha r^{2}} \quad$ Gaussian

$\emptyset(r)=\sqrt{r^{2}+c^{2}} \quad$ Multi-quadratic

$\varnothing(r)=(1-\varepsilon r)^{4}(4 \varepsilon r+1) \quad$ Wendland

where, $\alpha$ and $c$ are positive constants and $r \geq 0$.

Let given values of known centers are $(-3,-0.5,1.8,15$, 16), the known values of unknown function are $(5,3,-6,12$, 40). Unknown values of the function in the working space $(-32$, 32) were computed in one-dimensional space to show the behaviors of the kernel functions. According to RBF kernels, obtained results are given in Fig. 1.

Here, linear kernel result is not given since its behavior is well-known. When Fig. 1 is examined carefully, thin-plate spline kernel generates smooth curve. As mentioned in previous published studies, thin-plate spline kernel is wellsuited for cranial defects [6], [16].
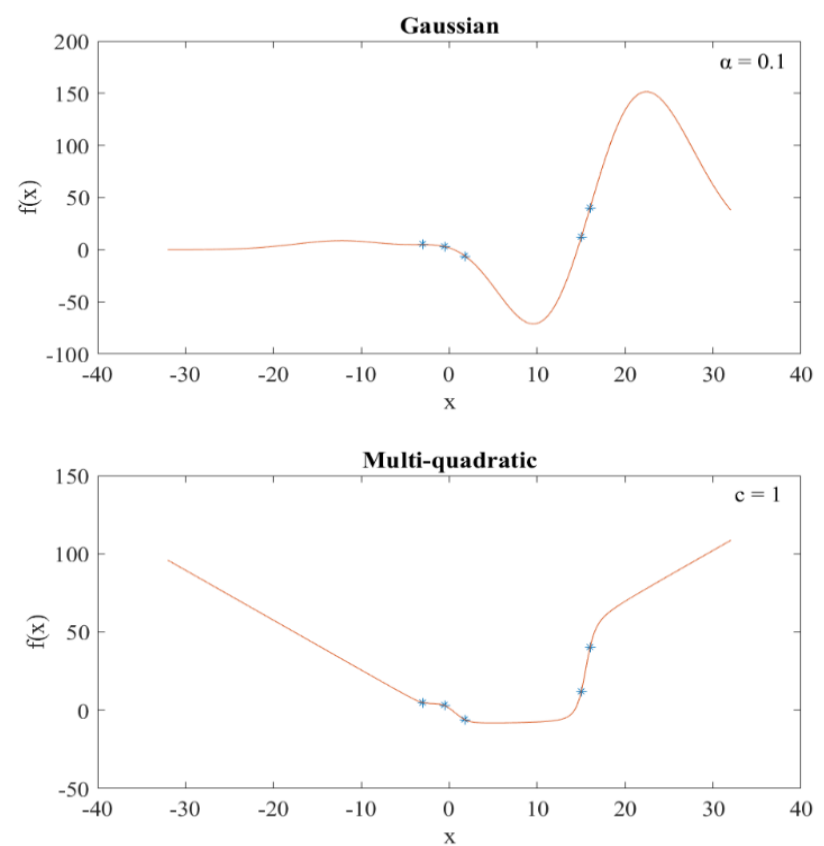
Carr et al. published their study about RBF interpolation for cranioplasty applications. According to the study, RBF interpolation method is superior then parametric spline interpolants, tensor product spline interpolants, etc. Even with large missing region, $\mathrm{RBF}$ has variable characterizations that make them well-suited for scattered data interpolation. However, we stated in our previous study [16] that scattered data interpolation generated good results by using RBF if large defected region is not on elliptical regions of a skull. The elliptical region of the skull is given in Fig. 2. When the diameter of defected region on elliptical region grows (bigger than $13 \mathrm{~mm}$ ), generated results deteriorate. Thereby, we propose that RBF interpolation performance in cranioplasty applications can be improved with using symmetrical data information.

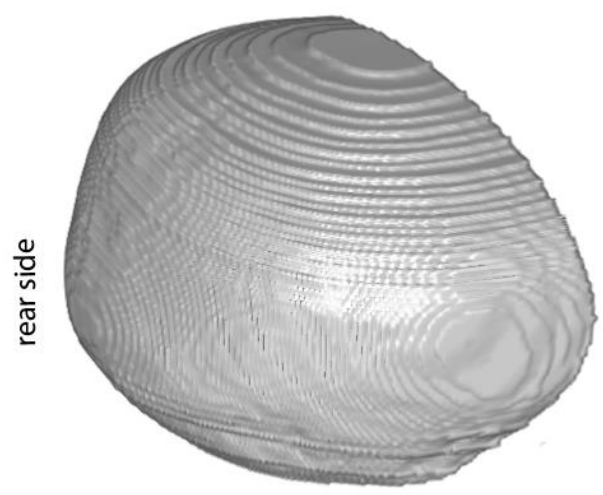

Fig. 2. Elliptical region of a skull.

The method which we implemented in our previous study is described below:

- Scattered data is obtained from CT images.

- Skull is segmented (soft tissue and background is removed).

- Normal vector of defected region is calculated by using its neighbors.

- A plane is created $d \mathrm{~mm}$ away from the defected region in the normal vector direction (green plane in Fig. 3).

- A height map is created by sending rays (red lines in Fig. 3) from the green plane to the skull.

- Distances of the rays that touch the skull surface (blue region in Fig. 3) are determined as RBF known values of center points.

- Distances of the rays that do not touch the skull surface are determined as defected region.

- $\lambda$ and polynomial coefficients are calculated for known center points.

- The distances of unknown points (defected region) are computed by RBF method with $\lambda$ and polynomial coefficients.

- For the defected region, voxel values are updated according to the computed distances.

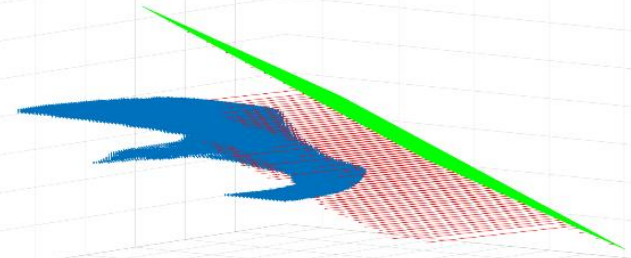

Fig. 3. Calculating the distances between plane and scattered data.

For thickness of the cement based implants, rays that touch the skull is continued along the bone. Thus, thickness map of the implant is calculated.

Fig. 4 shows 3D calculated height map of the defected region and its neighbors. Non-significant data on the height map was deleted before visualization.

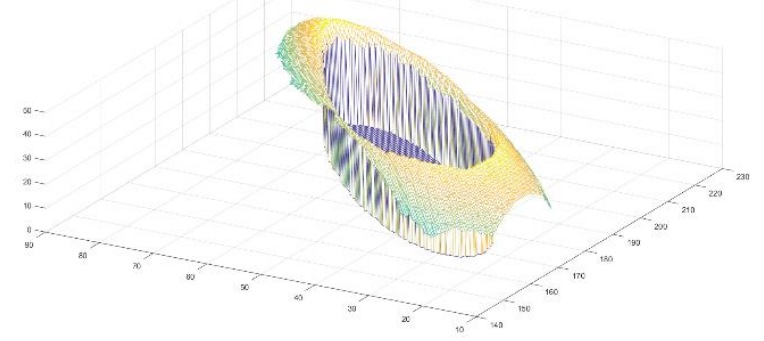

Fig. 4. 3D calculated height map of the defected reigon and its neighbours.

Weakness of the defined method comes up with large defects, because healthy neighbors of the defected region remain far away from the center of large defects. Therefore, computed distances become non-acceptable for the defected region. Hereby, symmetrical data of the defected region can be used to improve RBF performance. Axial view of the ineffective surface interpolation example is shown in Fig. 5. Bold red region in Fig. 5(a) denotes original skull while bold green region denotes the symmetrical data in Fig. 5(b) and thin white line demonstrates surface interpolation result in Fig. 5(a), (b) and (c).

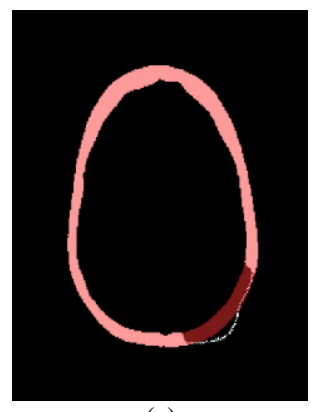

(a)

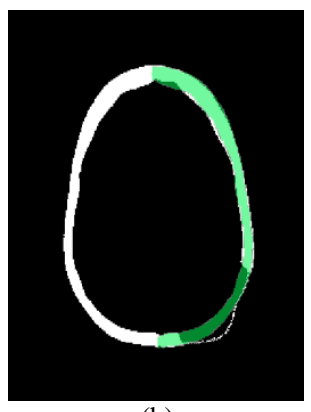

(b)

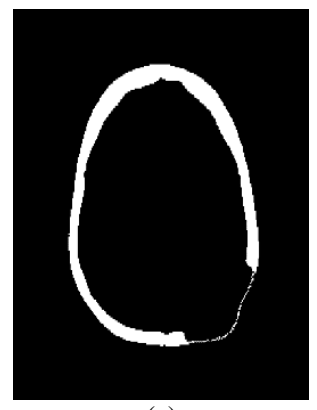

(c)
Fig. 5. Surface interpolation results (a) compared with original skull, (b) compared with symmetrical data, (c) RBF interpolation result.

For this improvement, we suggest that symmetrical data of the defected region can be used as healthy neighbors. Thus, maximum error is limited by using this new approach. In this approach, computed height map is updated with symmetrical data. In Fig. 6, black regions show non-significant data for scattered data interpolation, white region is the defected part of 
the skull, grey region represents height values of healthy neighbors, and grey dots in white region demonstrates height values sampled from symmetrical side.

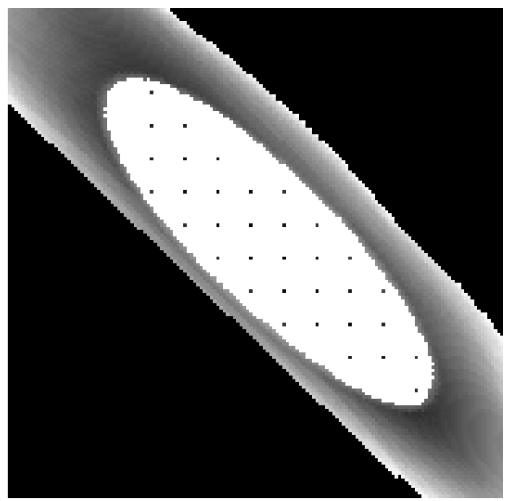

Fig. 6. The 2D projection of the height map.

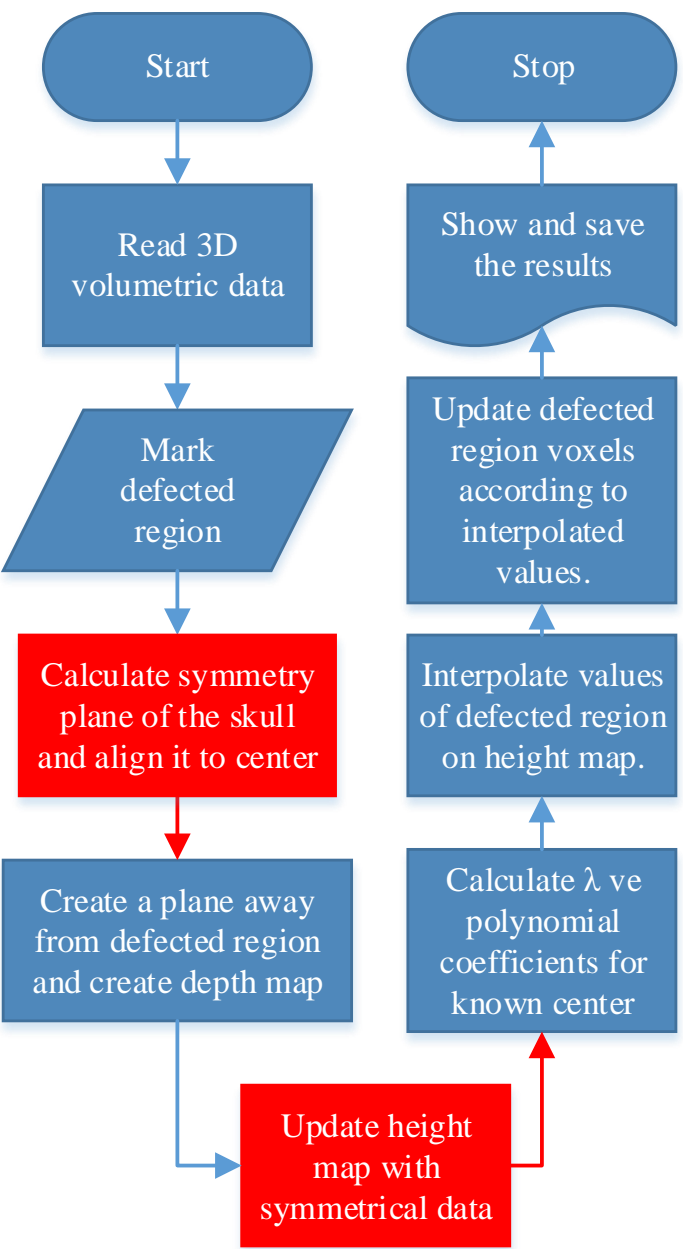

Fig. 7. Flowchart of the previously proposed method (blue boxes) and suggested improvements (red boxes).

The mirrored symmetrical side of the skull is replaced with the defected side. Then, rays are sent periodically from the green plane (Fig. 3) to the skull for the defected region. Thus, sampled distances of the symmetrical data are observed. At the end, the sampled distances are $1 \mathrm{~mm}$ shortened for the implant to stay outside of the defected region.

Modified flowchart of the new approach is shown in Fig. 7. In the figure, previously proposed method (blue boxes) and suggested improvement (red boxes) are given.

\section{RESULTS AND DISCUSSIONS}

The CT image used in this study is the same in our previous study with $1 \mathrm{~mm}$ slice spacing and 512 x 512 resolution. The difference of $7 \mathrm{~mm}$ between the computed implant model and the original skull in the previous study was reduced to $2 \mathrm{~mm}$ with the proposed new approach. Diameter of the defected region was $13 \mathrm{~mm}$ in the tests. Intensity-based mutual information was used for symmetry plane computation. Defected region was marked in 3D Slicer and the marked region was used as a mask. Defected region and its symmetry were not considered during computation.

Results of the proposed method were compared with previous studies [6], [16]. Achievement of scattered data interpolation with $\mathrm{RBF}$ for cranioplasty applications was improved by using the symmetrical data. The differences between previously proposed method and new approach are shown in detail in Fig. 8 and 9. If Fig. 8 is compared carefully, it can be seen that new approach improved the success of scattered data interpolation. The computed data on the outer surface is close to the original data. Thus, this achievement will provide a great advantage in terms of aesthetics, when the social and psychological conditions of the patients are considered, although the new approach has a disability on inner surface as shown in Fig. 9(c).

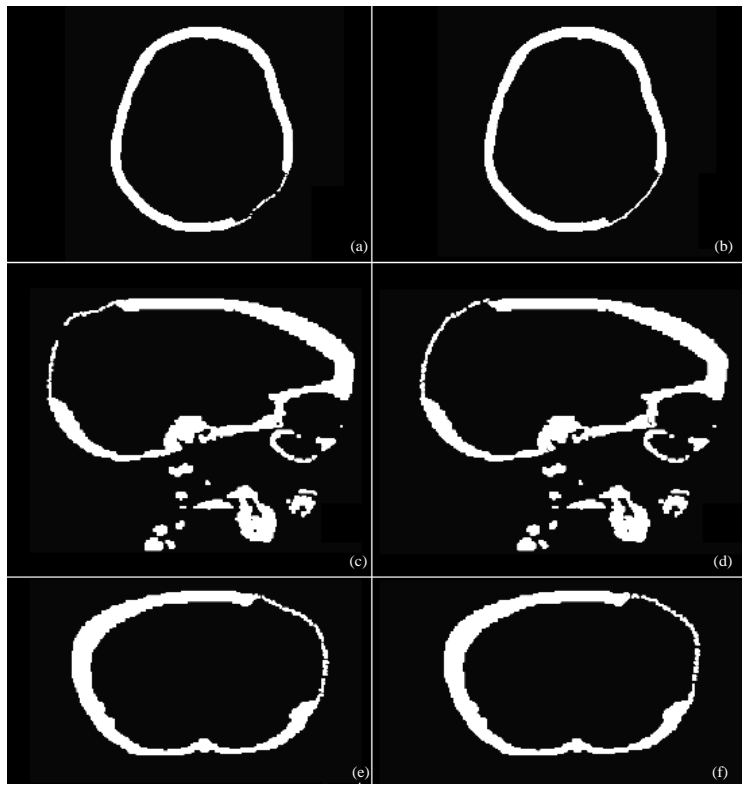

Fig. 8. Result comparison. The left side shows previously presented study results and the right side shows new approach results. (a) and (b) are axial slices, (c) and (d) are sagital slices, (e) and (f) are coronal slices.

Since the thickness of the implant can be disregarded for high-strength materials such as titanium, the results of new approach will be feasible. When cement-based materials are used, the thickness of the implant becomes important. 
Therefore, the inner surface of the results should be improved for the implants which are manufactured by 3D printers. Obtained results for inner surface is given in Fig. 9.

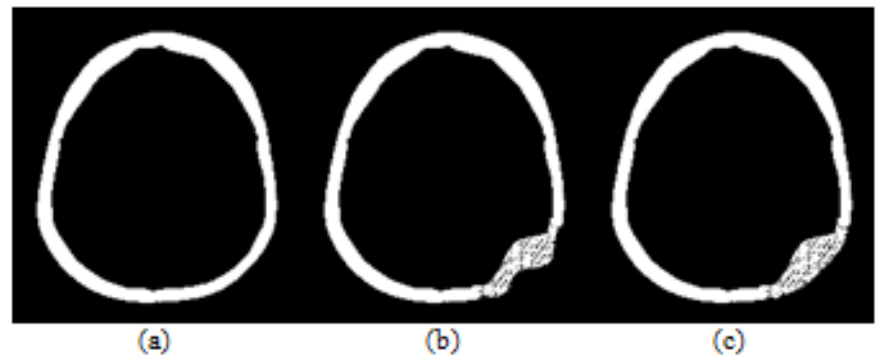

Fig. 9. Axial slices (a) Original data, (b) previously proposed method, (c) new approach.

It is already known that the previous presented method [16] is successful in tests which the defect size is small and thinplate spline kernel is the optimal RBF kernel as given in the previous study. If upside of a skull is defected, even linear kernel results are successful. Therefore, large defects on elliptical region (parietal-occipital bones) are focused in this study. The 3D results of previously proposed method and new approach are shown in Fig. 10.

The new approach searches symmetrical data to improve $\mathrm{RBF}$ performance. If the defected region does not have symmetrical data, it is not crucial, scattered data interpolation is realized by previously proposed method.

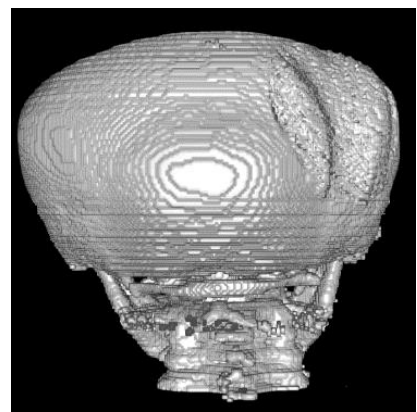

(a)

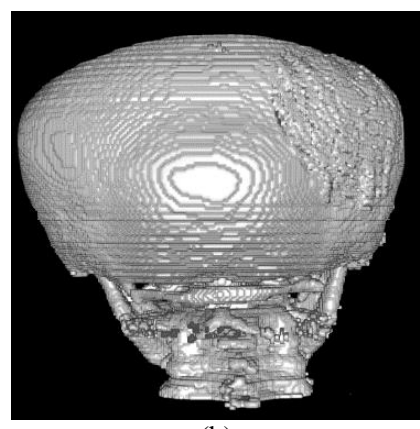

(b)
Fig. 10. The computed results, (a) previously proposed method, (b) new approach.

\section{CONCLUSION}

In this study, RBF interpolation was improved for cranial implant design. Defected part on outer surface of a skull can be completed using RBF and symmetrical data for cranioplasty. As a result, difference between the computed implant model in previous study [16] and the original skull was reduced to $2 \mathrm{~mm}$ with the newly-proposed approach. In addition, removal of aesthetic concerns is a great success when the sociological and psychological conditions of patients are considered. Nevertheless, there is a problem for inner surfaces of skulls to minimize errors. Therefore, a new approach should be enhanced for cement-based implants which can be manufactured with $3 \mathrm{D}$ printers. In next study, inner surface interpolation errors will be minimized and the thickness of cement based implants will be similar to original.

\section{REFERENCES}

[1] S. Aydin, B. Kucukyuruk, B. Abuzayed, S. Aydin, and G. Z. Sanus, "Cranioplasty: Review of materials and techniques.," J. Neurosci. Rural Pract., vol. 2, no. 2, pp. 162-167, 2011.

[2] S. Chibbaro et al., "Decompressive craniectomy and early cranioplasty for the management of severe head injury: A prospective multicenter study on 147 patients," World Neurosurg., vol. 75, no. 3-4, pp. 558-562, 2011.

[3] S. Honeybul and K. M. Ho, "Long-term complications of decompressive craniectomy for head injury.," J. Neurotrauma, vol. 28, no. 6, pp. 929935, 2011.

[4] R. M. Redfern and H. Pülhorn, "Cranioplasty," Adv Clin Neurosci Reabil., vol. 7, no. 5, pp. 32-34, 2007.

[5] Q. Yu et al., "Skull repair materials applied in cranioplasty: History and progress," Transl. Neurosci. Clin., vol. 3, no. 1, pp. 48-57, 2017.

[6] J. C. Carr, W. R. Fright, and R. K. Beatson, "Surface interpolation with radial basis functions for medical imaging," IEEE Trans. Med. Imaging, vol. 16, no. 1, pp. 96-107, 1997.

[7] E. Heissler et al., "Custom-made cast titanium implants produced with $\mathrm{CAD} / \mathrm{CAM}$ for the reconstruction of cranium defects.," Int. J. Oral Maxillofac. Surg., vol. 27, no. 5, pp. 334-338, 1998.

[8] M. Lee, C. Chang, C. Lin, L. Lo, and Y. Chen, "Three-Dimensional Image Reconstruction and Rapid Prototyping Models Improve Defect Evaluation, Treatment Planning, Implant Design, and Surgeon Accuracy," Eng. Med. Biol., no. April, 2002.

[9] H. Fu, L. Gao, L. Ju, and Y. Liu, "Personalized Cranium Defects Restoration Technique Based on Reverse Engineering," Tsinghua Sci. Technol., vol. 14, no. SUPPL. 1, pp. 82-88, 2009.

[10] N. Gerber, L. Stieglitz, M. Peterhans, L.-P. Nolte, A. Raabe, and S. Weber, "Using rapid prototyping molds to create patient specific polymethylmethacrylate implants in cranioplasty," in 2010 Annual International Conference of the IEEE Engineering in Medicine and Biology, 2010, pp. 3357-3360.

[11] W. A. Y. W. Yusoff, H. H. M. Ali, and M. A. H. M. Shukri, "Fabrication of surgical cranioplasty biomodel using fused deposition modeling," ICIMTR 2012 - 2012 Int. Conf. Innov. Manag. Technol. Res., pp. 550$554,2012$.

[12] W.-M. Kung, S.-T. Chen, C.-H. Lin, Y.-M. Lu, T.-H. Chen, and M.-S. Lin, "Verifying Three-Dimensional Skull Model Reconstruction Using Cranial Index of Symmetry," PLoS One, vol. 8, no. 10, p. e74267, Oct. 2013.

[13] J. Castelan, L. Schaeffer, A. Daleffe, D. Fritzen, V. Salvaro, and F. Pinto, "Manufacture of custom-made cranial implants from DICOM ® images using 3D printing , CAD / CAM technology and incremental sheet forming," vol. 30, no. 1996, pp. 265-273, 2014.

[14] M. Rudek, Y. B. Gumiel, and O. Canciglieri, "Autonomous ct replacement method for the skull prosthesis modelling," Facta Univ. Ser. Mech. Eng., vol. 13, no. 3, pp. 283-294, 2015.

[15] W. J. Van Der Meer, "3D Workflows in Orthodontics , Maxillofacial Surgery and Prosthodontics," 2016.

[16] F. Atasoy, F. Nar, B. Sen, and M. Ferat, "Scattered data estimation on medical images for cranioplasty applications," in 22nd Signal Processing and Communications Applications Conference (SIU), 2014, pp. 16821685.

[17] V. Skala, "Fast reconstruction of corrupted images and videos by radial basis functions," 2013 Int. Conf. Control. Autom. Inf. Sci. ICCAIS 2013, pp. 267-271, 2013.

[18] W. Wang, "An Image Inpainting Algorithm Based on CSRBF Interpolation,” Int. J. Inf. Technol., vol. 28, pp. 112-119, 2006.

[19] M. Alsalamah and S. Amin, "Medical Image Inpainting with RBF Interpolation Technique," Int. J. Adv. Comput. Sci. Appl., vol. 7, no. 8, pp. 112-119, 2016. 